\title{
Homocysteine induces mitochondrial dysfunction and oxidative stress in myocardial ischemia/reperfusion injury through stimulating ROS production and the ERK1/2 signaling pathway
}

\author{
LEI WANG, HEPING NIU and JUN ZHANG \\ Department of Cardiology, Cangzhou Central Hospital, Cangzhou, Hebei 061001, P.R. China
}

Received January 29, 2019; Accepted January 06, 2020

DOI: $10.3892 /$ etm.2020.8735

\begin{abstract}
Acute oxidative stress and mitochondrial dysfunction are crucial for acute myocardial ischemia-reperfusion (AMI/R) injury, which may induce cell or mitochondrial membrane rupture and myocardial infarction. Plasma homocysteine (Hcy) expression levels are positively associated with risk of cardiovascular disease, and ERK1/2 exert anti-apoptotic and cardioprotective effects on AMI/R injury. However, the precise molecular mechanism of action underlying the effects of Hcy and the ERK1/2 signaling pathway on mitochondrial dysfunction and oxidative stress in $\mathrm{AMI} / \mathrm{R}$ injury remains unclear. In the present study, AMI/R injury models were established in an animal model treated with Hcy and in H9C2 cells that were treated with hypoxia-reoxygenation. Mitochondrial function and oxidative stress were evaluated. The results demonstrated that Hcy enhanced ERK1/2 protein expression levels and oxidative stress, induced cytochrome $\mathrm{c}$ translocation and mitochondria dysfunction, and caused cardiac dysfunction in rats with AMI/R injury. However, an ERK1/2 inhibitor effectively protected AMI/R injury rats from Hcy-induced cardiac dysfunction and oxidative stress. In conclusion, Hcy induced mitochondrial dysfunction and oxidative stress in AMI/R injury through stimulating ROS production and the ERK1/2 signaling pathway. An ERK1/2 inhibitor may be an effective new therapeutic method for treating Hcy-induced cardiac dysfunction in patients with $\mathrm{AMI} / \mathrm{R}$ injury.
\end{abstract}

\section{Introduction}

Coronary artery obstruction presenting with acute myocardial infarction is one of the leading causes of death worldwide (1). Ischemia-reperfusion (I/R) injury, a pervasive pathological disorder, occurs when the blood flow to a tissue is blocked and

Correspondence to: Dr Jun Zhang, Department of Cardiology, Cangzhou Central Hospital, 16 Xinhua West Road, Cangzhou, Hebei 061001, P.R. China

E-mail: junzhang159@163.com

Key words: ERK1/2, homocysteine, ischemia-reperfusion, mitochondrial dysfunction, oxidative stress reperfusion occurs when the blockage is removed (2-4). Acute myocardial I/R (AMI/R) injury is a complex phenomenon involving atherosclerotic plaque rupture (5), which stops the oxygen and nutrient supply, inducing cardiomyocyte death due to the reperfusion process. Previous studies have demonstrated that mitochondrial activities play a critical role in the early phase of AMI/R $(6,7)$.

Reactive oxygen species (ROS) from mitochondria drive the acute damage in reperfusion (8), which directly causes mitochondrial ATP disruption (9), calcium level dysregulation (10) and leads to cell apoptosis (8). The acute oxidative stress injury and mitochondrial dysfunction are important steps for AMI/R injury, which can induce cell or mitochondrial membrane rupturing and myocardial infarction. The major source of oxidative stress in I/R injury may be associated with complex I and III of the electron transport chain in mitochondria (11). Therefore, alterations in the activity of complex I and complex III in mitochondria may influence the oxidative damage caused by ROS.

The ERK1/2 signaling pathway is one of the important pathways that utilize proteins from the mitogen-activated protein kinases family, which exert anti-apoptotic and cardioprotective effects during AMI/R (12). However, the extent of ERK phosphorylation is significantly raised in $\mathrm{I} / \mathrm{R}$ injury. Previous studies have demonstrated that ERK protects against I/R injury through activating p90 ribosomal S6 kinase and phosphorylation of $\mathrm{Bcl}-\mathrm{xl} / \mathrm{Bcl}-2$-associated proteins to inhibit cellular apoptosis $(13,14)$. The ERK1/2 signaling pathway has been identified as the pro-survival mediator against I/R injury. PD98059 is a highly selective inhibitor of ERK which binds to the inactive form of the protein and prevents activation by its upstream activators (15). PD98059 may act against ERK signal transduction and therefore act as a powerful tool to investigate the mechanisms of action behind I/R injury.

Homocysteine (Hcy), the sulfur-containing amino acid form during methionine metabolism, which is associated with the risk of I/R (16). Abnormally high levels of Hcy in plasma have been indicated as a strong and independent risk factor for cardiovascular disease (17). Recent studies have reported that Hcy plays a critical role in I/R-induced oxidative stress, which causes the activation of inflammatory pathways, impaired endothelial function, atherogenesis, and cardiac remodeling (17). Furthermore, plasma Hcy expression levels 
are also positively associated with blood pressure, a major risk factor for cardiovascular disease $(18,19)$.

However, the precise effects of Hcy and the ERK1/2 signaling pathway on mitochondrial dysfunction and oxidative stress in AMI/R injury remains unclear. In the present study, AMI/R injury animal models were established and treated with Hcy. Hcy was also used in H9C2 cells that were subjected to hypoxia-reoxygenation $(\mathrm{H} / \mathrm{R})$. Mitochondrial function and ROS production were evaluated.

\section{Materials and methods}

Animal model of AMI/R. Male Sprague-Dawley rats $(250 \pm 10 \mathrm{~g})$ were purchased from Vital River Laboratory Co. Ltd. The animal care and experimental procedures were according to the Guide for the Care and Use of Laboratory Animals published by the National Institutes of Health. The study protocol was reviewed and approved by the Ethics Committee of Cangzhou Central Hospital (approval no. 2018-029-01). Rats were maintained in environmentally controlled rooms $\left(25^{\circ} \mathrm{C}\right)$ with $12 \mathrm{~h}$ light/dark cycles. Rats were randomly divided into 4 experimental groups with 5 rats per group: Sham operation control group, AMI/R group, AMI/R and Hcy group (AMI/R + Hcy), and the AMI/R, Hcy and PD98059-treated group (AMI/R + Hcy + PD98059). Rats were sacrificed 21 days after reperfusions. Blood samples and heart tissue were collected to measure the cardiac I/R-related protein expression.

The AMI/R rat model was established according to a previous study (20). In brief, ischemia was maintained for $30 \mathrm{~min}$, at which time the slip knot was released, initiating reperfusion for $3 \mathrm{~h}$. After intraperitoneal injection of $2 \%$ pentobarbital $(30 \mathrm{mg} / \mathrm{kg})$, the left anterior descending coronary artery was ligated for $45 \mathrm{~min}$ before reperfusion injury in the AMI/R group. To prevent infection, benzyl penicillin sodium (400,000 U/kg) was injected once a day for 3 days. Successful AMI/R was confirmed by ST segment-characterized electrocardiogram. The Hcy (1.6 mg/kg/day; Sigma-Aldrich; Merck $\mathrm{KGaA}$ ) was injected intravenously via the tail vein for 21 days prior to the operation and up to $24 \mathrm{~h}$ after surgery. In the AMI/R + Hcy + PD98059 group, ERK1/2 inhibitor PD98059 $(10 \mathrm{mg} / \mathrm{kg}$ ) was administered throughout the entire period of Hcy treatment in the AMI/R rats. All rats were euthanized following the AVMA Guidelines on Euthanasia (21) after the experiment. Intraperitoneal injections of a minimum of $200 \mathrm{mg} / \mathrm{kg}$ sodium pentobarbital were used as the euthanasia solution. Following the completion of the euthanasia procedure, death was confirmed by ascertaining cardiac and respiratory arrest or noting an animal's fixed and dilated pupils.

Cell culture and the I/R model. H9C2 (2-1) cells (CRL-1446 ${ }^{\mathrm{TM}}$ ) were obtained from the American Type Culture Collection and cultured in DMEM containing 10\% FBS (Gibco; Thermo Fisher Scientific, Inc.), $2 \mathrm{mM}$ glutamine, $100 \mathrm{U} / \mathrm{ml}$ of penicillin and $100 \mu \mathrm{g} / \mathrm{ml}$ of streptomycin and exposed to $\mathrm{H} / \mathrm{R}$ conditions as previously described (22). The H/R treatments consisted of $0.1 \% \mathrm{O}_{2}+5 \% \mathrm{CO}_{2}$ in serum-starvation FBS medium for $4 \mathrm{~h}$. After hypoxia, the cells were re-oxygenated by incubating in $95 \% \mathrm{O}_{2}+5 \% \mathrm{CO}_{2}$ environment. Then, $\mathrm{H} 9 \mathrm{C} 2$ cells were randomly exposed to one of the following treatments:
Pretreated ERK inhibitor PD98059 $(50 \mu \mathrm{M})$ for $30 \mathrm{~min}+\mathrm{Hcy}$ $(500 \mu \mathrm{M})$ or Hcy $(500 \mu \mathrm{M})$ only at $37^{\circ} \mathrm{C}$.

Western blotting. Rat normal or ischemic heart tissues were homogenized in RIPA buffer $(20 \mathrm{mM}$ TRIS-HCl $\mathrm{pH} \mathrm{7.5,}$ $150 \mathrm{mM} \mathrm{NaCl}, 1 \mathrm{mM}$ EDTA, $1 \%$ Triton-X100, 1\% sodium deoxycholate, $1 \mathrm{mM}$ PMSF and $1 \mu \mathrm{g} / \mathrm{ml}$ leupeptin). The concentration of the protein was determined using Lowry protein assay and equal amounts $(20 \mu \mathrm{g})$ of protein were separated by $10 \%$ SDS-PAGE. After transferring onto nitrocellulose membranes, the membranes were blocked for $1 \mathrm{~h}$ with $5 \%$ non-fat milk at $25^{\circ} \mathrm{C}$ and then incubated with the following primary antibodies: Phosphorylated (p)-p38 (1:1,000; Abcam; cat. no. ab4822), p38 (1:2,000; Abcam; cat. no. ab170099), VDAC1/porin (1:2,000; Abcam; cat. no. ab14734), p-ERK (1:1,000; Abcam; cat. no. ab192591), ERK1/2 (1:5,000; Abcam; cat. no. ab184699), peroxisome proliferator activated receptor $\gamma$ (PPAR $\gamma ; 1: 2,000$; Abcam; cat. no. ab45036), p53 (1:5,000; Santa Cruz Biotechnology, Inc.; cat. no. sc-126), cytochrome c (1:1,000; Abcam; cat. no. ab133504), cleaved caspase 3 (1:2,000; Santa Cruz Biotechnology, Inc.; cat. no. sc-56053) and GAPDH (1:5,000; Santa Cruz Biotechnology, Inc.; cat. no. sc-32233) at $4{ }^{\circ} \mathrm{C}$ overnight. The membrane was incubated with the corresponding secondary antibody (goat anti-rabbit or goat anti-mouse; 1:10,000; Abcam; cat. no. ab6702 or ab6708, respectively) at $25^{\circ} \mathrm{C}$ and then analyzed under a fluorescence microscope. The protein levels were quantified using ImageJ 1.48 (National Institute of Health).

Determination of cellular and mitochondrial ROS. The formation of ROS in $\mathrm{H} 9 \mathrm{C} 2$ cells was measured using the fluorescein dye, dichloro-dihydro-fluorescein diacetate (DCF-DA). The cells were seeded into six-well plates $\left(5 \times 10^{5}\right)$ and pre-incubated with $10 \mu \mathrm{M}$ DCF-DA in the dark. The fluorescence of DCF-DA was then detected at the excitation wavelength of $488 \mathrm{~nm}$ and emission wavelength of $525 \mathrm{~nm}$. Mitochondrial ROS production was measured using the mitochondrial superoxide indicator, MitoSOX ${ }^{\mathrm{TM}}$ (cat. no. LSM36008; Invitrogen; Thermo Fisher Scientific, Inc.) according to the manufacturer's instruction. MitoSOX was added to the cells and incubated for $10 \mathrm{~min}$ at $37^{\circ} \mathrm{C}$ after the various treatments. Subsequently, cells were washed and detected using confocal microscopy imaging.

Measurement of heart function. Following $2 \mathrm{~h}$ after reperfusion, creatine kinase $(\mathrm{CK})$ and glutamic-oxaloacetic transaminase (GOT) levels were determined using an automatic biochemistry analyzer (Toshiba model 750; Toshiba Corporation) following the manufacturer's instructions. Left ventricular systolic pressure (LVSP), maximum change rate of left ventricular pressure rise $\left(+\mathrm{dp} / \mathrm{dt}_{\max }\right)$ and the maximum change rate of left ventricular pressure fall (-dp/dt $\mathrm{dmax}_{\text {mal }}$ were recorded using echocardiography (Vivid E95; GE Healthcare) following the manufacturer's protocol.

Statistical analysis. All data are presented as the mean \pm SEM. Statistical analyses were performed using one-way ANOVAs followed by post-hoc Tukey's test for multiple comparisons in GraphPad Prism (GraphPad Software, Inc.). $\mathrm{P}<0.05$ was considered to indicate a statistically significant difference. 


\section{Results}

Hcy enhances ERK1/2 protein expression and oxidative stress in rats with $A M I / R$ injury. ERK1/2 expression was analyzed in a rat model of AMI/R injury. The western blot analysis showed significant differences between the AMI/R and AMI/R + Hcy groups, revealing that the protein expression ratio of p-ERK1/2/total-ERK1/2 was increased after Hcy treatment (Fig. 1A). To determine whether Hcy was capable of inducing oxidative stress in AMI/R injury, AMI/R rats were treated with Hcy + PD98059 or Hcy alone, and the concentration of ROS was assessed by DCF-DA. The results showed that the $\mathrm{AMI} / \mathrm{R}+$ Hcy treatment resulted in a significant enhancement in ROS generation as compared to the AMI/R group (Fig. 1B). These results demonstrated that the protein expression of ERK1/2 was enhanced and that oxidative stress was induced after administration of Hcy in rats with AMI/R injury.

Hcy induces cytochrome $c$ translocation and mitochondrial dysfunction in rats with $A M I / R$ injury. To explore the effects of Hcy on AMI/R-injury induced mitochondria-mediated apoptosis, the protein expression levels of cytochrome c were investigated by western blotting. Significantly decreased expression levels of mitochondrial cytochrome $\mathrm{c}$ were detected in the $\mathrm{AMI} / \mathrm{R}+\mathrm{Hcy}$ group as compared to the AMI/R group (Fig. 1A). By contrast, compared with the $\mathrm{AMI} / \mathrm{R}$ group, a significant increase in the protein expression levels of cytochrome $\mathrm{c}$ in the cytosolic fraction was observed in the AMI/R + Hcy group (Fig. 1A). To determine whether Hcy induced mitochondrial dysfunction in the AMI/R injury rats, the mitochondrial oxidative damage was evaluated. Immunofluorescence staining showed that the number of MitoSOX-positive cells was significantly higher in the AMI/R + Hcy group compared with the AMI/R group (Fig. 1B). The data revealed that Hcy significantly enhanced the release of mitochondrial cytochrome $\mathrm{c}$ into the cytosol and increased ROS generation from mitochondria in AMI/R injury rats.

Hcy causes cardiac dysfunction in rats with AMI/R injury. Using cardiac dysfunction analysis, the current study determined the effects of Hcy on AMI/R injury-induced cardiac dysfunction. Compared with the AMI/R group, Hcy significantly decreased the LVSP and $+\mathrm{dp} / \mathrm{dt}_{\max }$. However, the $-\mathrm{dp} / \mathrm{dt}_{\max }$ has been enhanced by Hcy (Fig. 2). Furthermore, the activity of CK and GOT was significantly increased by Hcy in AMI/R injury rats (Fig. 2).

ERK1/2 inhibitor reverses the effects of Hcy on mitochondrial dysfunction and oxidative stress in rats with AMI/R injury. To assess whether ERK1/2 was involved in the effects of Hcy on inducing mitochondrial dysfunction and oxidative stress, cellular ROS production and mitochondrial function was evaluated in rats with $\mathrm{AMI} / \mathrm{R}$ injury. A significant increase in mitochondrial cytochrome $\mathrm{c}$ expression levels was observed after PD98059 treatment as compared to the AMI/R injury rats following Hcy treatment (Fig. 1A). These data suggested that PD98059 treatment caused a suppressive effect on cellular and mitochondrial ROS production. Furthermore, PD98059 significantly inhibited the release of mitochondrial cytochrome $\mathrm{c}$ into the cytosol in the AMI/R + Hcy rats after Hcy treatment. As shown in Fig. 1B, PD98059 significantly inhibited ROS generation after Hcy treatment in AMI/R injury rats. Consistent with this observation, mitochondrial ROS production was also attenuated in the PD98059-treated animals as compared with the AMI/R + Hcy group (Fig. 1B).

ERK1/2 inhibitor reverses the effects of Hcy on inducing cardiac dysfunction in rats with AMI/R injury. Whether ERK1/2 was involved in the effects of Hcy on inducing cardiac dysfunction in rats with AMI/R injury was further evaluated. After ERK1/2 inhibitor PD98059 treatment, the effects of Hcy on LVSP, $+\mathrm{dp} / \mathrm{dt}_{\max }$ and $-\mathrm{dp} / \mathrm{dt}_{\max }$ were significantly reversed. In addition, the activity of both CK and GOT was significantly decreased by PD 98059 treatment in the AMI/R injury rats following Hcy treatment (Fig. 2).

ERK1/2 inhibitor reverses the effects of Hcy on mitochondrial dysfunction and cell injury in $H / R H 9 C 2$ cells. To verify the beneficial effects of ERK1/2 inhibitor on Hcy-induced cardiac dysfunction, $\mathrm{H} 9 \mathrm{C} 2$ cells were exposed to $\mathrm{H} / \mathrm{R}$ conditions and treated with or without Hcy or PD98059. Afterco-treatment with Hcy and H/R, the p-ERK1/2/ERK1/2 ratio was significantly increased compared to the H/R group (Fig. 3A). Subsequently, the rate of apoptosis was determined by western blotting and annexin-V staining assay. Compared with the H/R group, the proportion of annexin- $\mathrm{V}$ positive cells and the expression of p53 were significantly increased (Fig. 3A and B); however, the levels of p-p38/total p38 and PPAR $\gamma$ were significantly decreased in the H/R + Hcy group (Fig. 3A). Pretreatment with PD98059 could reverse the Hcy-induced increase in the levels of apoptosis in $\mathrm{H} 9 \mathrm{C} 2$ cells (Fig. 3A and B). Similar results were observed using DCF-DA fluorescence. The H/R + Hcy + PD98059 group demonstrated significantly reduced oxidative stress compared with that in the H/R + Hcy group (Fig. 4). The data revealed that the ERK1/2 inhibitor could prevent the Hcy-induced apoptosis and oxidative stress.

To confirm the findings in vitro, the effects of ERK1/2 inhibitor on Hcy-stimulated mitochondrial dysfunction were also examined in $\mathrm{H} 9 \mathrm{C} 2$ cells. As shown in Fig. 3A, the mitochondrial cytochrome $\mathrm{c}$ levels were significantly increased in the H/R + Hcy + PD98059 group compared with the H/R + Hcy group. Consistent with the in vivo data, the mitochondrial ROS production was suppressed following PD98059 treatment, compared with the AMI/R + Hcy group (Fig. 4). Taken together, these results demonstrated that the ERK1/2 inhibitor decreased ROS generation and suppressed cell apoptosis, thereby exerting a protective role in Hcy-induced cardiac dysfunction in $\mathrm{H} 9 \mathrm{C} 2$ cells.

\section{Discussion}

A number of studies have shown that coronary heart disease is a major cause of death and disability worldwide (23). Coronary heart disease is usually associated with the detrimental effects of AMI/R (5). I/R not only appears in different organs but is also involved in various pathological processes, such as heart failure. Previous studies have shown that the apoptosis of cardiomyocytes is the most important pathogenic mechanisms behind AMI/R injury $(24,25)$. Reperfusion injury after ischemia is characterized by myocardial stunning, myocyte death and microvascular dysfunction. The mechanisms of action behind 

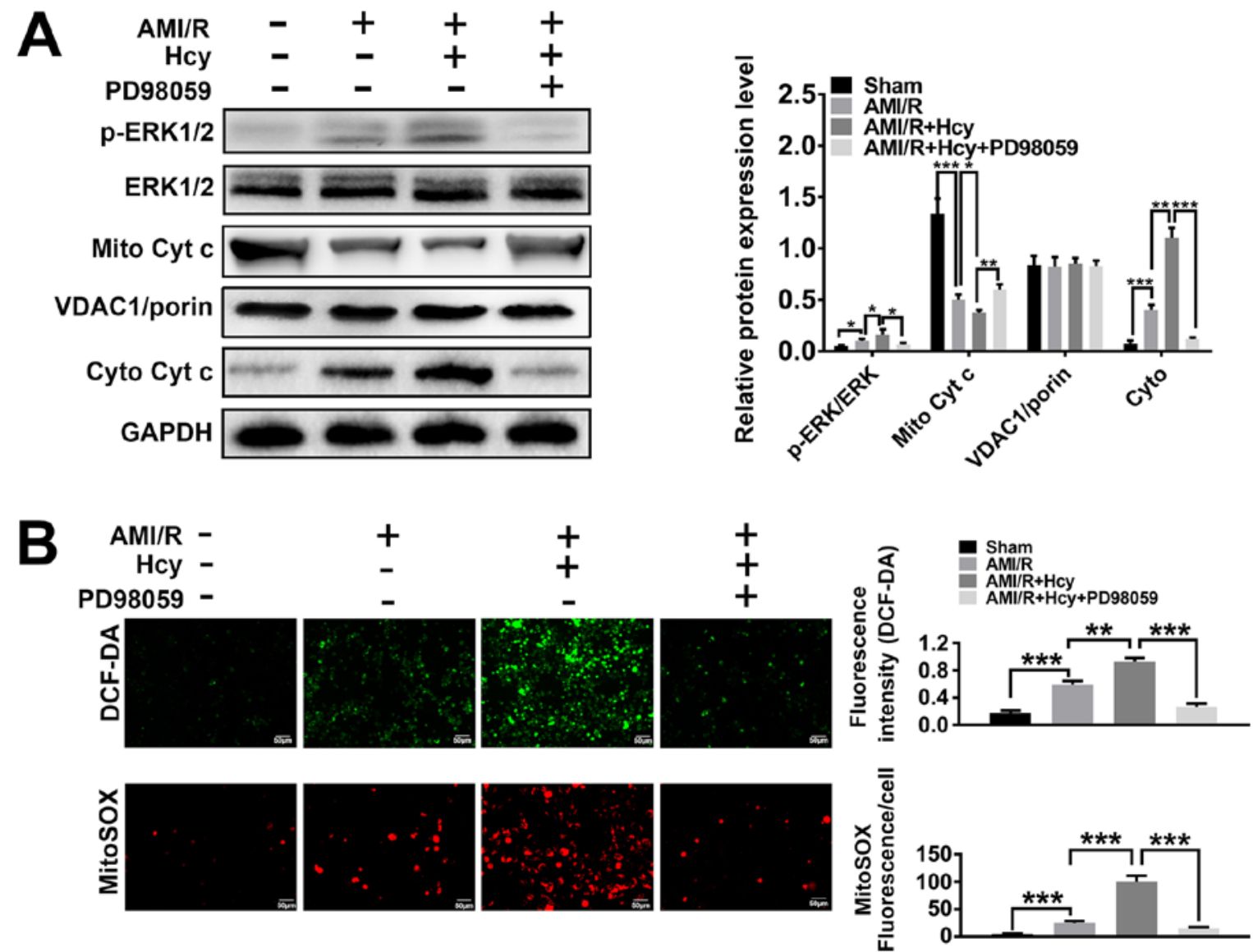

Figure 1.ERK1/2 inhibitor PD98059 reverses the effects of Hcy on ERK 1/2 expression, the cytoplasmic translocation of Cyt c and cellular and mitochondrial ROS production in rats with AMI/R injury. (A) Western blotting for ERK and Cyt $\mathrm{c}$ after Hcy treatment. Bar graphs show semi-quantitative analysis of the levels of ERK and Cyt c, as determined by band density analysis ( $\mathrm{n}=5)$. (B) Reactive oxygen species production in the cell matrix and mitochondria after Hcy treatment in rats with AMI/R. Representative images of the green DCF-DA or red MitoSOX staining $(\mathrm{n}=5) .{ }^{*} \mathrm{P}<0.05,{ }^{* *} \mathrm{P}<0.01,{ }^{* * *} \mathrm{P}<0.005$. AMI/R, acute myocardial ischemia/reperfusion; Cyto, cytoplasmic; Cyt c, cytochrome c; DCF-DA, dichloro-dihydro-fluorescein diacetate; Hcy, DL-homocysteine; Mito, mitochondrial; p, phosphorylated.


Figure 2. ERK1/2 inhibitor PD98059 reverses the effects of Hcy on inducing cardiac dysfunction in rats with AMI/R injury. PD98059 ameliorated the changes in LVSP, $+\mathrm{dp} / \mathrm{dt}_{\max }$ and $-\mathrm{dp} / \mathrm{dt}_{\max }$ in the rats with AMI/R injury. Bar graphs show semi-quantitative analysis of the levels of CK and GOT as determined by an automatic biochemistry analyzer, as well as the LVSP, $+\mathrm{dp} / \mathrm{dt}_{\max }$ and $-\mathrm{dp} / \mathrm{dt}_{\max }$, as determined by echocardiography. $\mathrm{n}=5 ;{ }^{*} \mathrm{P}<0.05$ and ${ }^{* * *} \mathrm{P}<0.01$. AMI/R, acute myocardial I/R; CK, creatine kinase; GOT, glutamic-oxaloacetic transaminase; LVSP, left ventricular systolic pressure; Hcy, D,L-homocysteine; +dp/dt ${ }_{\text {max }}$, the maximum change rate of left ventricular pressure rise; - $\mathrm{dp} / \mathrm{dt}_{\max }$, the maximum change rate of left ventricular pressure fall.

$\mathrm{AMI} / \mathrm{R}$ remain complex. Recent advances have indicated that oxidative stress, mitochondrial membrane depolarization, calcium overloading and inflammation are all involved. There are numerous kinases and signaling pathway involved in I/R-induced cell apoptosis. Activation of pro-survival kinases, such as the PI3K-Akt and ERK1/2, have been shown to be critical in AMI/R-induced cardioprotection (26). Hcy plays a critical role in the metabolism of sulfur amino acids and is associated with cardiovascular vascular disorders (27). The auto-oxidation process of Hcy is highly reactive at the physiological $\mathrm{pH}$ and leads to the production of superoxide and hydrogen peroxide (28). This phenomenon indicates that ROS production from the auto-oxidation of Hcy remains one of the mechanisms of action contributing to Hcy-induced cell injury. A previous study reported that increasing Hcy expression levels in plasma may enhance smooth muscle cell proliferation and collagen production, resulting in vascular disease (29). However, the effects and mechanisms of action behind Hcy induced cellular injury in AMI/R have not yet been fully elicited. Considering that ERK1/2 pathway activation, oxidative 

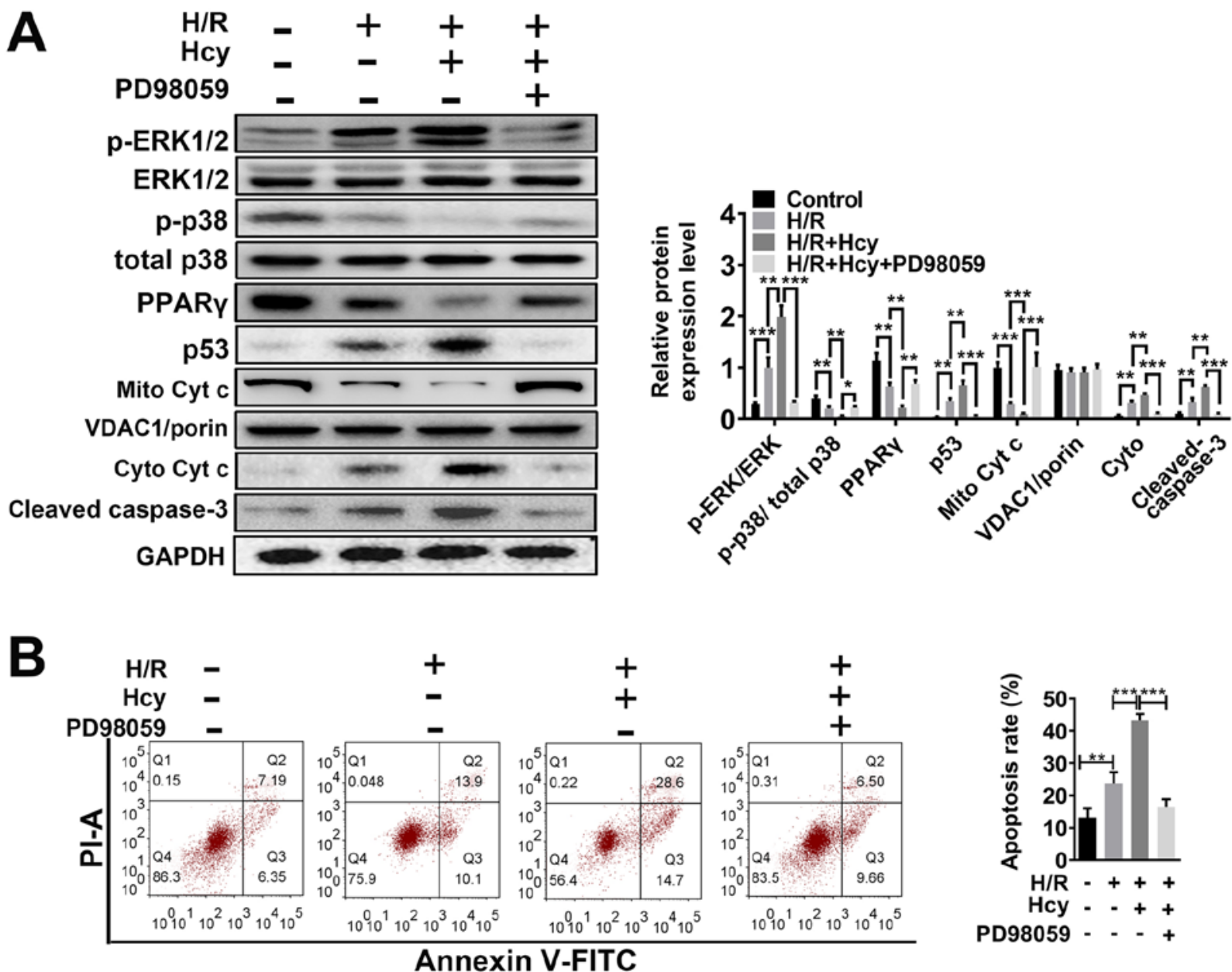

Figure 3. ERK1/2 inhibitor PD98059 reverses the effects of Hcy on inducing Cyt c cytoplasmic translocation and apoptosis in H/R H9C2 cells. (A) Western blotting was performed to determine the expression levels of ERK and apoptosis-related proteins. Bar graphs show semiquantitative analysis of the levels of p/total ERK, p-p38, PPAR $\gamma$, p53, cleaved caspase 3 and Cyt c, as determined by band density analysis. (B) The apoptosis rate was evaluated using Annexin V-FITC and PI staining, using an imaging flow cytometer. ${ }^{*} \mathrm{P}<0.05,{ }^{* *} \mathrm{P}<0.01,{ }^{* * *} \mathrm{P}<0.005$. Cyto, cytoplasmic; Cyt c, cytochrome c; Hcy, DL-homocysteine; H/R, hypoxia-reoxygenation; Mito, mitochondrial; p, phosphorylated; PI; propidium iodide; PPAR $\gamma$, peroxisome proliferator activated receptor $\gamma$.
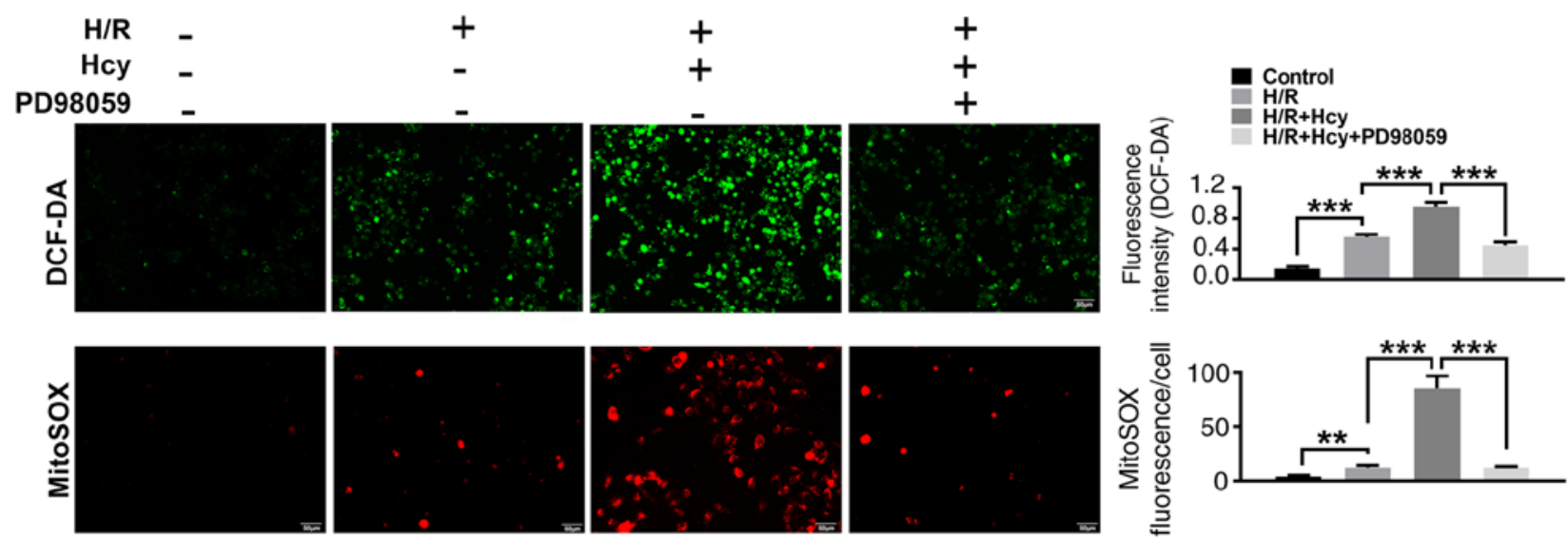

Figure 4. ERK1/2 inhibitor PD98059 reverses the effects of Hcy on cellular and mitochondrial ROS production in H/R H9C2 cells. Representative images of green DCF-DA or red MitoSOX staining are shown. ROS production in the cell matrix and mitochondria in H/R H9C2 cells after Hcy treatment are calculated from the fluorescence intensities. ${ }^{* *} \mathrm{P}<0.01$ and ${ }^{* * *} \mathrm{P}<0.005$. DCF-DA, dichloro-dihydro-fluorescein diacetate; ROS, reactive oxygen species; Hcy, DL-homocysteine.

stress and mitochondrial dysfunction all play a critical role in the process of AMI/R injury, the present study analyzed the functional relevance of these factors in Hcy-induced cell injury in AMI/R. The results of the present study showed that after Hcy treatment in AMI/R rats, ERK1/2 prosphorylation and oxidative stress were significantly elevated. Hcy also enhanced 
the release of mitochondrial cytochrome $\mathrm{c}$ into the cytosol and increased the ROS generation from mitochondria in $\mathrm{AMI} / \mathrm{R}$ rats. These results are in accordance with previous research which indicated that the role of Hcy in endothelial dysfunction is mediated by oxidative stress and inflammation (30). Furthermore, the LVSP, $+\mathrm{dp} / \mathrm{dt}_{\max }$ and $-\mathrm{dp} / \mathrm{dt}_{\max }$, as well as the activity of $\mathrm{CK}$ and GOT were all significantly increased by Hcy during AMI/R injury. These data are consistent with previous studies which reported that Hcy may be involved in cardiovascular diseases through a number of mechanisms and that Hcy may alter arterial structure and function $(31,32)$.

As the ERK1/2 signaling pathway is known to regulate the inflammatory processes in cardiovascular disease, the ERK1/2 signaling pathway may become a new therapeutic target for heart failure $(33,34)$. To further explore the significance of the ERK1/2 signaling pathway in cardioprotection during Hcy treatment in AMI/R, the ERK inhibitor, PD98059, was used to investigate the role of the ERK1/2 signaling pathway in Hcy-induced cell injury. The present results indicated that the ERK1/2 inhibitor not only protected I/R injury rats from Hcy-induced mitochondrial dysfunction and oxidative stress but also improved the myocardial function following Hcy-induced cardiac dysfunction. Furthermore, in the cell model, the inhibition of ERK1/2 also decreased ROS generation and apoptosis, thereby suggesting a protective effect against Hcy-induced cardiac dysfunction in $\mathrm{H} 9 \mathrm{C} 2$ cells.

In conclusion, the present study demonstrated that the protective effect of the ERK1/2 inhibitor could reverse the Hcy-induced cellular injury. ERK1/2 inhibitors may be a new therapeutic method to treat Hcy-induced cardiac dysfunction in $\mathrm{AMI} / \mathrm{R}$.

\section{Acknowledgements}

Not applicable.

\section{Funding}

No funding was received.

\section{Availability of data and materials}

The datasets used and/or analyzed during the current study are available from the corresponding author on reasonable request.

\section{Authors' contributions}

LW, HN and JZ performed the experiments and wrote the manuscript, collected and analyzed the experimental data, revised the manuscript, designed the experiments and approved the final version manuscript.

\section{Ethics approval and consent to participate}

The study protocol was reviewed and approved by the Ethics Committee of Cangzhou Central Hospital (no. 2018-029-01).

\section{Patient consent for publication}

Not applicable.

\section{Competing interests}

The authors declare that they have no competing interests.

\section{References}

1. Thygesen K, Alpert JS, Jaffe AS, Simoons ML, Chaitman BR, White HD; Joint ESC/ACCF/AHA/WHF Task Force for Universal Definition of Myocardial Infarction; Authors/Task Force Members Chairpersons, Thygesen K, Alpert JS, et al: Third universal definition of myocardial infarction. J Am Coll Cardiol 60: 1581-1598, 2012.

2. Hausenloy DJ and Yellon DM: Myocardial ischemia-reperfusion injury: A neglected therapeutic target. J Clin Invest 123: 92-100, 2013.

3. Eltzschig HK and Eckle T: Ischemia and reperfusion-from mechanism to translation. Nat Med 17: 1391, 2011.

4. Yellon DM and Hausenloy DJ: Myocardial reperfusion injury. N Engl J Med 357: 1121-1135, 2007.

5. Heusch G, Libby P, Gersh B, Yellon D, Böhm M, Lopaschuk G and Opie L: Cardiovascular in coronary artery disease and heart failure. Lancet 383: 1933-1943, 2014.

6. Ganote C, Worstell J and Kaltenbach J: Oxygen-induced enzyme release after irreversible myocardial injury. Effects of cyanide in perfused rat hearts. Am J Pathol 84: 327-350, 1976.

7. Zweier JL, Flaherty JT and Weisfeldt ML: Direct measurement of free radical generation following reperfusion of ischemic myocardium. Proc Natl Acad Sci USA 84: 1404-1407, 1987.

8. Loor G, Kondapalli J, Iwase H, Chandel NS, Waypa GB, Guzy RD, Vanden Hoek TL and Schumacker PT: Mitochondrial oxidant stress triggers cell death in simulated ischemia-reperfusion. Biochim Biophys Acta 1813: 1382-1394, 2011.

9. Murphy E and Steenbergen C: Mechanisms underlying acute protection from cardiac ischemia-reperfusion injury. Physiol Rev 88: 581-609, 2008.

10. Di Lisa F and Bernardi P: Modulation of mitochondrial permeability transition in ischemia-reperfusion injury of the heart. Advantages and limitations. Curr Med Chem 22: 2480-2487, 2015.

11. Paradies G, Petrosillo G, Pistolese M and Ruggiero FM: Reactive oxygen species affect mitochondrial electron transport complex I activity through oxidative cardiolipin damage. Gene 286: 135-141, 2002.

12. Abe Ji, Baines CP and Berk BC: Role of mitogen-activated protein kinases in ischemia and reperfusion injury: The good and the bad. Circ Res 86: 607-609, 2000.

13. Das A, Salloum FN, Xi L, Rao YJ and Kukreja RC: ERK phosphorylation mediates sildenafil-induced myocardial protection against ischemia-reperfusion injury in mice. Am J Physiol Heart Circ Physiol 296: H1236-H1243, 2009.

14. Yue TL, Wang C, Gu JL, Ma XL, Kumar S, Lee JC, Feuerstein GZ, Thomas H, Maleeff B and Ohlstein EH: Inhibition of extracellular signal-regulated kinase enhances ischemia/reoxygenation-induced apoptosis in cultured cardiac myocytes and exaggerates reperfusion injury in isolated perfused heart. Circ Res 86: 692-699, 2000.

15. Di Cristo G, Berardi N, Cancedda L, Pizzorusso T, Putignano E, Ratto GM and Maffei L: Requirement of ERK activation for visual cortical plasticity. Science. 292: 2337-2340, 2001.

16. Wald DS, Law M and Morris JK: Homocysteine and cardiovascular disease: Evidence on causality from a meta-analysis. BMJ 325: 1202, 2002.

17. Ganguly P and Alam SF: Role of homocysteine in the development of cardiovascular disease. Nutri J 14: 6, 2015.

18. Malinow M, Levenson J, Giral P, Nieto F, Razavian M, Segond P and Simon A: Role of blood pressure, uric acid, and hemorheological parameters on plasma homocyst (e)ine concentration. Atherosclerosis 114: 175-183, 1995.

19. Hoogeveen EK, Kostense PJ, Beks PJ, Mackaay AJ, Jakobs C, Bouter LM, Heine RJ and Stehouwer CD: Hyperhomocysteinemia is associated with an increased risk of cardiovascular disease, especially in non-insulin-dependent diabetes mellitus: A population-based study. Arterioscler Thromb Vasc Biol 18: 133-138, 1998.

20. Shao L, Wu D, Zhang P, Li W, Wang J, Su G, Liao Y, Wang Z and Liu K: The significance of microthrombosis and fgl2 in no-reflow phenomenon of rats with acute myocardial ischemia/reperfusion. Clin Appl Thromb Hemost 19: 19-28, 2013. 
21. Leary S, Underwood W, Anthony R, Cartner S, Corey D, Grandin T, Greenacre C, Gwaltney-Brant S, McCrackin MA, Meyer R, et al: AVMA guidelines for the euthanasia of animals: 2013 edition.

22. Yin Y, Guan Y, Duan J, Wei G, Zhu Y, Quan W, Guo C, Zhou D, Wang Y, Xi M and Wen A: Cardioprotective effect of Danshensu against myocardial ischemia/reperfusion injury and inhibits apoptosis of H9c2 cardiomyocytes via Akt and ERK1/2 phosphorylation. Eur J Pharmacol 699: 219-226, 2013.

23. Feigin VL, Forouzanfar MH, Krishnamurthi R, Mensah GA, Connor M, Bennett DA, Moran AE, Sacco RL, Anderson L, Truelsen T, et al: Global and regional burden of stroke during 1990-2010: Findings from the Global Burden of Disease Study 2010. Lancet 383: 245-255, 2014.

24. Scarabelli TM, Stephanou A, Pasini E, Comini L, Raddino R, Knight RA and Latchman DS: Different signaling pathways induce apoptosis in endothelial cells and cardiac myocytes during ischemia/reperfusion injury. Circ Res 90: 745-748, 2002 .

25. Xu Q, Li X, Lu Y, Shen L, Zhang J, Cao S, Huang X, Bin J and Liao Y: Pharmacological modulation of autophagy to protect cardiomyocytes according to the time windows of ischaemia/reperfusion. Br J Pharmacol 172: 3072-3085, 2015.

26. Kim SJ, Yoo KY, Jeong CW, Kim WM, Lee HK, Bae HB, Kwak SH, Li M and Lee J: Urinary trypsin inhibitors afford cardioprotective effects through activation of PI3K-Akt and ERK signal transduction and inhibition of p38 MAPK and JNK. Cardiology 114: 264-270, 2009.

27. Hassan A, Hunt BJ, O'sullivan M, Bell R, D'souza R, Jeffery S, Bamford JM and Markus HS: Homocysteine is a risk factor for cerebral small vessel disease, acting via endothelial dysfunction. Brain 127: 212-219, 2004.
28. Misra HP: Generation of superoxide free radical during the autoxidation of thiols. J Biol Chem 249: 2151-2155, 1974.

29. Majors A, Ehrhart LA and Pezacka EH: Homocysteine as a risk factor for vascular disease. Enhanced collagen production and accumulation by smooth muscle cells. Arterioscler Thromb Vasc Biol 17: 2074-2081, 1997

30. Basu A, Jenkins AJ, Stoner JA, Thorpe SR, Klein RL, Lopes-Virella MF, Garvey WT, Lyons TJ and DCCT/EDIC Research Group: Plasma total homocysteine and carotid intima-media thickness in type 1 diabetes: A prospective study. Atherosclerosis 236: 188-195, 2014.

31. Zhang S, Bai YY,Luo LM, Xiao WK, Wu HM and Ye P: Association between serum homocysteine and arterial stiffness in elderly: A community-based study. J Geriatr Cardiol 11: 32-38, 2014.

32. Balakumar P, Singh AP and Singh M: Rodent models of heart failure. J Pharmacol Toxicol Methods 56: 1-10, 2007.

33. Purcell NH, Wilkins BJ, York A, Saba-El-Leil MK, Meloche S, Robbins $\mathrm{J}$ and Molkentin JD: Genetic inhibition of cardiac ERK1/2 promotes stress-induced apoptosis and heart failure but has no effect on hypertrophy in vivo. Proc Natl Acad Sci USA 104: 14074-14079, 2007.

34. Bueno OF, De Windt LJ, Tymitz KM, Witt SA, Kimball TR, Klevitsky R, Hewett TE, Jones SP, Lefer DJ, Peng CF, et al: The MEK1-ERK1/2 signaling pathway promotes compensated cardiac hypertrophy in transgenic mice. EMBO J 19: 6341-6350, 2000

(i) $($ This work is licensed under a Creative Commons Attribution-NonCommercial-NoDerivatives 4.0 International (CC BY-NC-ND 4.0) License. 